CRYSTALLOGRAPHIC COMMUNICATIONS

ISSN 2056-9890

\section{Crystal structure and Hirshfeld surface analysis of rac-2-[2-(4-chlorophenyl)-3,4-dihydro-2H-1-benzo- pyran-4-ylidene]hydrazine-1-carbothioamide}

\author{
Ruokuosenuo Zatsu, ${ }^{a}$ Prabhakar Maddela, ${ }^{a} *$ M. Indira Devi, ${ }^{a}$ Ranjit Singh ${ }^{\mathrm{b}}$ and \\ Chullikkattil P. Pradeep ${ }^{b}$
}

Received 11 February 2019

Accepted 13 April 2019

Edited by P. Bombicz, Hungarian Academy of Sciences, Hungary

Keywords: crystal structure; flavanone; chromane; thiosemicarbazide; Schiff base; $\mathrm{N}-\mathrm{H} \ldots \mathrm{S}$ hydrogen bonds; supramolecular chemistry; Hirshfeld surface analysis.

CCDC reference: 1893434

Supporting information: this article has supporting information at journals.iucr.org/e

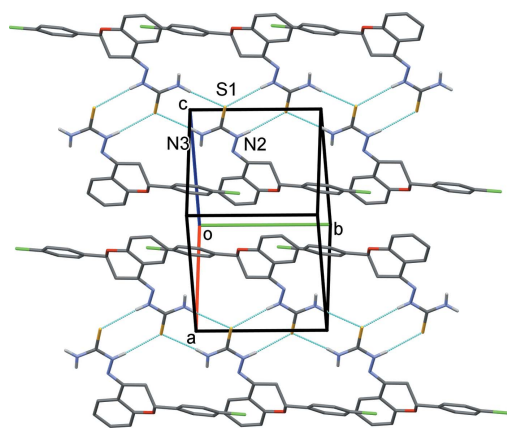

OPEN $\odot$ ACCESS

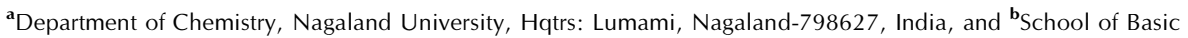
Sciences, Indian Institute of Technology Mandi, Mandi-175005, Himachal Pradesh, India. *Correspondence e-mail: prabha7chem@gmail.com

In the title compound, $\mathrm{C}_{16} \mathrm{H}_{14} \mathrm{~N}_{3} \mathrm{OSCl}$, a Schiff base derivative of a thiosemicarbazide with a flavanone, the 4-chlorophenyl ring is inclined to the benzene ring of the chromane ring system by $30.72(12)^{\circ}$. The pyran ring has an envelope conformation with the methine $\mathrm{C}$ atom as the flap. The mean plane of the thiourea unit is twisted with respect to the benzene ring of the chromanone ring system, subtending a dihedral angle of $19.78(19)^{\circ}$. In the crystal, molecules are linked by two pairs of $\mathrm{N}-\mathrm{H} \cdots \mathrm{S}$ hydrogen bonds, forming inversion dimers enclosing $R_{2}^{2}(8)$ ring motifs, which are linked to form ribbons propagating along the $b$-axis direction. The intermolecular contacts in the crystal have been analysed using Hirshfeld surface analysis.

\section{Chemical context}

Flavanones, a subclass of flavonoids, are widely recognized for their nutraceutical values (Testai \& Calderone, 2017). Flavanones are also known for their potential bioactivities against cancer (Bauvois et al., 2003). Thiosemicarbazides are a class of versatile ligands exhibiting important physicochemical properties due to their $\pi$-delocalization and flexibility of coordination modes. Therefore, a combination of flavanones and thiosemicarbazides may lead to compounds having synergistic properties of both classes of compounds. Schiff base derivatives of thiosemicarbazides have been studied for their biological and pharmacological properties (Bai et al., 2017). However, Schiff base derivatives of flavanones with thiosemicarbazides have not been explored extensively (Brodowska et al., 2016; Bargujar et al., 2018). In particular, structurally characterized flavanone-thiosemicarbazone Schiff bases are rare in the literature. The presence of $\mathrm{NH}$ and $\mathrm{S}$ moieties in such compounds opens up the possibility of studying the role of the comparatively less explored class of $\mathrm{N}-\mathrm{H} \cdots \mathrm{S}$ interactions in building supramolecular architectures. This is of interest as hydrogen bonding to sulfur is known to play an important role in biological systems (Andersen et al., 2014; Walters et al., 2005). Considering the above, we have synthesized the title compound through a Schiff base condensation reaction, and report herein on its crystal structure and the Hirshfeld surface analysis. 


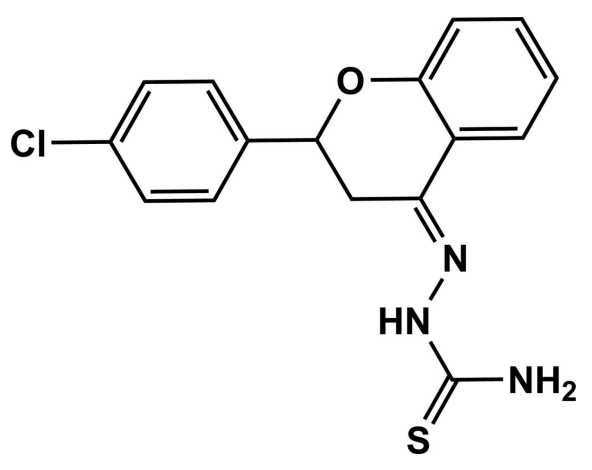

\section{Structural commentary}

The molecular structure of the title compound is illustrated in Fig. 1. The 4-chlorophenyl ring (C11-C16) is inclined to the benzene ring (C5-C10) of the chromanone ring system by $30.72(12)^{\circ}$. The pyran ring $(\mathrm{O} 1 / \mathrm{C} 2-\mathrm{C} 5 / \mathrm{C} 10)$ has an envelope conformation with atom $\mathrm{C} 2$ as the flap, being displaced by 0.655 (2) $\AA$ from the mean plane through the other five atoms of the ring. The mean plane of the thiourea unit $(\mathrm{N} 2 / \mathrm{C} 17 / \mathrm{S} 1 /$ $\mathrm{N} 3$ ) is twisted with respect to benzene ring (C5-C10) of the chromane ring system, forming a dihedral angle of $19.78(19)^{\circ}$.

\section{Supramolecular features}

A strong hydrogen bond often involves highly electronegative second row elements such as N, O and F. However, the less electronegative third row elements $(\mathrm{P}, \mathrm{S}$ and $\mathrm{Cl}$ ) are also known to take part in hydrogen-bonding interactions. In the crystal of the title compound, molecules are linked by two pairs of $\mathrm{N}-\mathrm{H} \cdots \mathrm{S}$ hydrogen bonds, forming inversion dimers

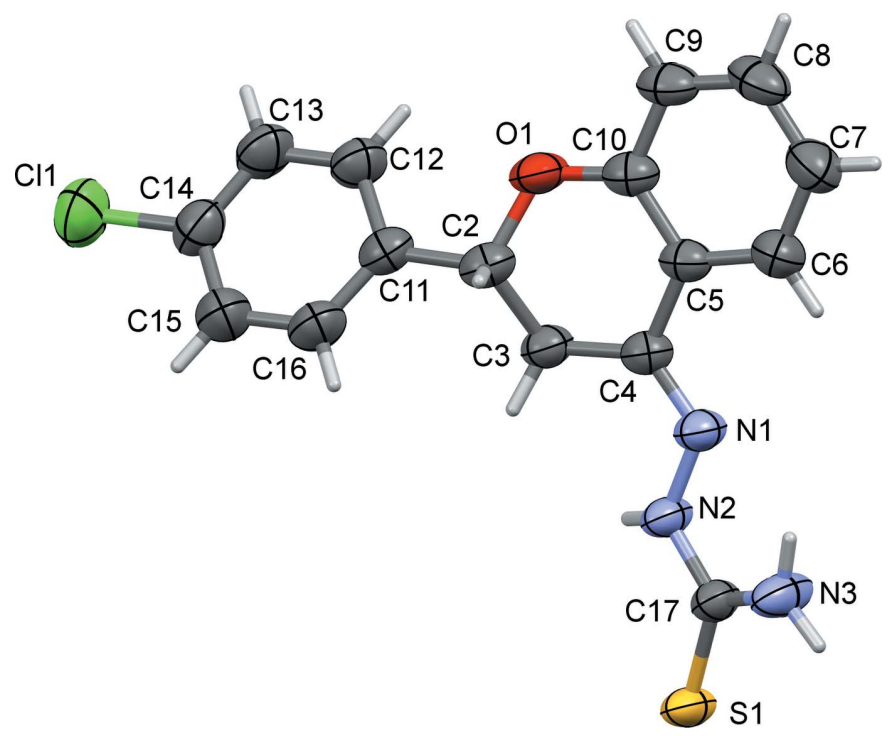

Figure 1

A view of the molecular structure of the title compound, with the atom labelling. Displacement ellipsoids are drawn at the $50 \%$ probability level. The orientation of the fiigure means that one of the two $\mathrm{H}$ atoms on $\mathrm{C} 3$ is not shown.
Table 1

Hydrogen-bond geometry $\left(\AA{ }^{\circ}\right)$.

\begin{tabular}{lllll}
\hline$D-\mathrm{H} \cdots A$ & $D-\mathrm{H}$ & $\mathrm{H} \cdots A$ & $D \cdots A$ & $D-\mathrm{H} \cdots A$ \\
\hline $\mathrm{N} 2-\mathrm{H} 2 N \cdots \mathrm{S} 1^{\mathrm{i}}$ & $0.85(3)$ & $2.65(3)$ & $3.480(2)$ & $167(2)$ \\
$\mathrm{N} 3-\mathrm{H} 3 B N \cdots \mathrm{S} 1^{\mathrm{ii}}$ & $0.88(3)$ & $2.52(3)$ & $3.392(2)$ & $171(2)$ \\
\hline
\end{tabular}

Symmetry codes: (i) $-x,-y+1,-z+2$; (ii) $-x,-y,-z+2$.

enclosing $R_{2}^{2}(8)$ ring motifs, which are linked to form ribbons propagating along the $b$-axis direction (Table 1 and Fig. 2). In the crystal, there are no other significant short intermolecular interactions present.

\section{Hirshfeld surface analysis and two-dimensional fingerprint plots for the title compound}

The Hirshfeld surface analysis (Spackman \& Jayatilaka, 2009) and the associated two-dimensional fingerprint plots (McKinnon et al., 2007) were performed with CrystalExplorer17 (Turner et al., 2017). A recent article by Tiekink and collaborators (Tan et al., 2019) 'outlines the various procedures and what can be learned by using CrystalExplorer'.

The Hirshfeld surface of the title compound mapped over $d_{\text {norm }}$ is given in Fig. 3a. The red spots indicate specific points of contact in the crystal. The Hirshfeld surface mapped over the shape-index is given in Fig. $3 b$, showing red spots and blue regions indicative of possible $\mathrm{C} \cdots \mathrm{H} / \mathrm{H} \cdots \mathrm{C}$ (i.e. $\mathrm{C}-\mathrm{H} \cdots \pi)$ contacts. The Hirshfeld surface mapped over the curvedness is given in Fig. $3 c$. Here the region around the chromane ring system is fairly flat, indicative of possible $\pi-\pi$ interactions. However, these interactions must be extremely weak as analysis of the structure using PLATON (Spek, 2009) did not

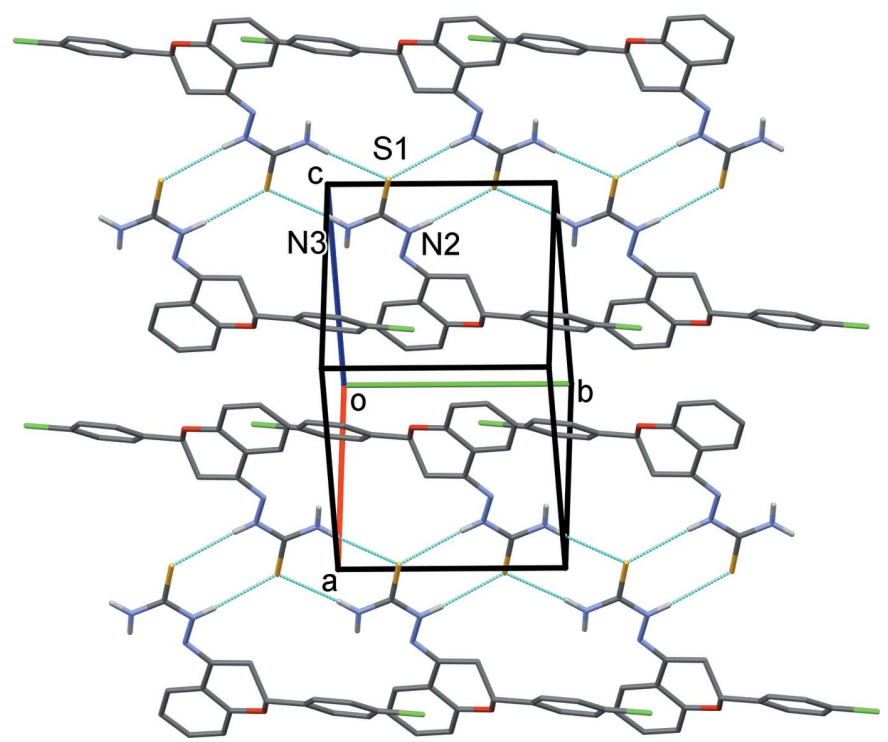

Figure 2

A view normal to plane (101) of the crystal packing of the title compound. The $\mathrm{N}-\mathrm{H} \cdots \mathrm{S}$ hydrogen bonds are shown as dashed lines (Table 1). For clarity, C-bound $\mathrm{H}$ atoms have been omitted. 

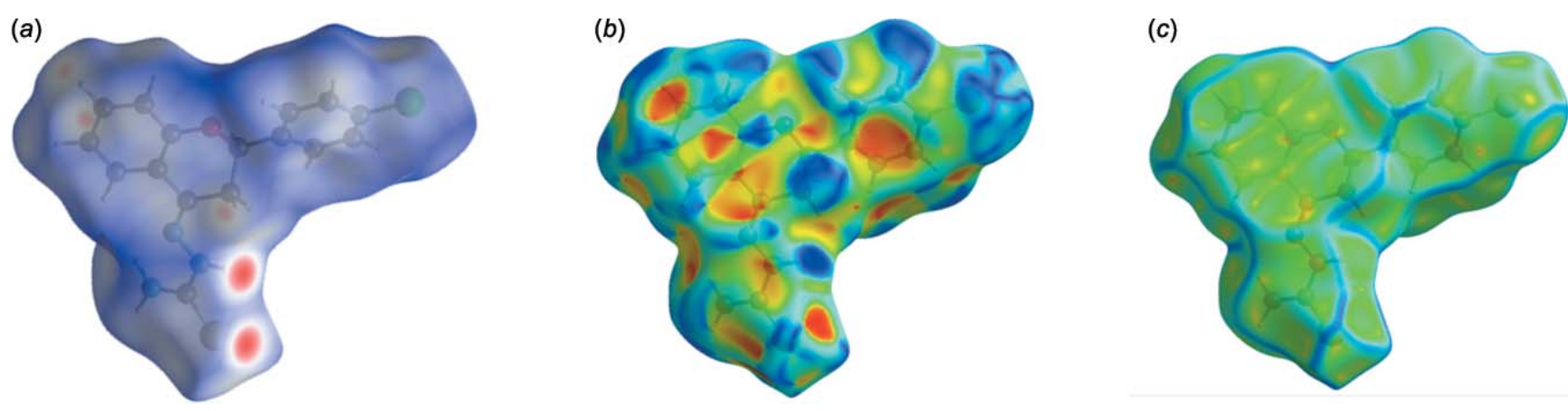

Figure 3

The Hirshfeld surface of the title compound mapped over $(a) d_{\text {norm }},-0.3525$ to 1.4929 arbitrary units, $(b)$ shape-index and $(c)$ curvedness.

indicate the presence of any significant $\mathrm{C}-\mathrm{H} \cdots \pi$ or offset $\pi-$ $\pi$ interactions in the crystal.

The full two-dimensional fingerprint plot for the title compound is given in Fig. 4a. The principal intermolecular interactions (Fig. $4 b-4 f)$ are delineated into $\mathrm{H} \cdots \mathrm{H}(38.9 \%)$, $\mathrm{C} \cdots \mathrm{H} / \mathrm{H} \cdots \mathrm{C}(20.3 \%), \mathrm{S} \cdots \mathrm{H} / \mathrm{H} \cdots \mathrm{S}(13.1 \%), \mathrm{Cl} \cdots \mathrm{H} / \mathrm{H} \cdots \mathrm{Cl}$ $(12.0 \%)$ and $\mathrm{N} \cdots \mathrm{H} / \mathrm{H} \cdots \mathrm{N}(3.0 \%)$ contacts. Note that only for the $\mathrm{H} \cdots \mathrm{H}, \mathrm{C} \cdots \mathrm{H} / \mathrm{H} \cdots \mathrm{C}$ and $\mathrm{S} \cdots \mathrm{H} / \mathrm{H} \cdots \mathrm{S}$ contacts is $d_{\mathrm{e}}+d_{\mathrm{i}}$ (where $d_{\mathrm{e}}$ and $d_{\mathrm{i}}$ are the distances from a given point on the surface to the nearest atom outside and inside, respectively), less than the sum of the van der Waals radii of the individual atoms.

\section{Database survey}

A search of the Cambridge Structural Database (CSD, Version 5.40, update February 2019; Groom et al., 2016) for a similar structure gave one hit, the compound $2^{\prime}$ [(2-(4-fluorophenyl)chroman-4-ylidene]isonicotinohydrazide

(CSD refcode TEJQUV; Nie et al., 2006). Here, the pyran ring has an envelope conformation and the 4-fluorophenyl ring is inclined to the benzene ring of the chromane ring system by $66.57(11)^{\circ}$. In the title compound, the pyran ring also has an envelope conformation and the 4-chloropheny ring is inclined
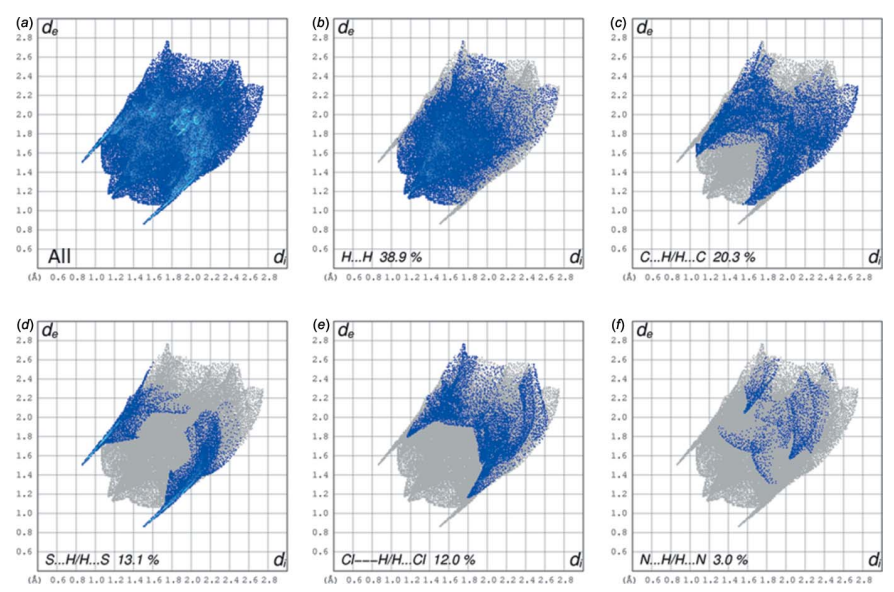

Figure 4

(a) The full two-dimensional fingerprint plot for the title compound and fingerprint plots delineated into $(b) \mathrm{H} \cdots \mathrm{H},(c) \mathrm{C} \cdots \mathrm{H} / \mathrm{H} \cdots \mathrm{C},(d) \mathrm{S} \cdots \mathrm{H} /$ $\mathrm{H} \cdots \mathrm{S},(e) \mathrm{Cl} \cdots \mathrm{H} / \mathrm{H} \cdots \mathrm{Cl}$ and $(f) \mathrm{N} \cdots \mathrm{H} / \mathrm{H} \cdots \mathrm{N}$ contacts. to the benzene ring of the chromane ring system by only $30.72(12)^{\circ}$.

A search for the 2-(tetrahydro-4H-pyran-4-ylidene)hydrazine-1-carbothioamide skeleton gave one hit, viz. (E)-2[2,6-bis(4-chlorophenyl)-3,5-dimethyltetrahydro-4H-pyran-4ylidene]hydrazinecarbothioamide (UQAWAL; Umamatheswari et al., 2011). Here, the pyran ring has a chair conformation and the bond lengths and angles of the hydrazinecarbothioamide unit are similar to those in the title compound.

\section{Synthesis and crystallization}

The synthesis of the title compound was achieved by following a reported procedure with some modifications (Bargale et al., 1988). Conc. $\mathrm{H}_{2} \mathrm{SO}_{4}(10 \mathrm{~mol} \%)$ in ethanol (5 ml) was added to a stirred solution of 2-(4-chlorophenyl)-chroman-4-one (0.258 g, $1 \mathrm{mmol}$ ) (Zheng et al., 2013) and thiosemicarbazide $(0.091 \mathrm{~g}, 1 \mathrm{mmol})$. The mixture was refluxed for $96 \mathrm{~h}$ with continuous stirring. After completion of the reaction, as monitored by TLC, the solvent was removed under reduce pressure and then ice-cold water was added. The resulting solid product was collected by filtration, washed with water (3-4 times) and finally with hexane and then dried at room temperature. Pale-yellow plate-like crystals of the title compound were obtained by slow evaporation at room temperature of a solution in acetonitrile (yield 90\%, m.p. 483$486 \mathrm{~K})$. IR ( $\left.\mathrm{KBr}, \mathrm{cm}^{-1}\right)$ : 3417, 3245, 3152, 2984, 2888, 2790, $1598,1512,1454,1298,1250,1089,1077,883,766,507,498 .{ }^{1} \mathrm{H}$ NMR $\left(400 \mathrm{MHz}, \mathrm{DMSO}-d_{6}\right), \delta$ ppm: $10.47(s, 1 \mathrm{H}, \mathrm{NH}), 8.32$ $\left(d, 2 \mathrm{H}, J=6.50 \mathrm{~Hz}, \mathrm{NH}_{2}\right) ; 8.13(s, 1 \mathrm{H}, \mathrm{Ar}-\mathrm{H}) ; 7.54(d d, 4 \mathrm{H}, J=$ $8.41 \mathrm{~Hz}, \mathrm{Ar}-\mathrm{H}) ; 7.35-7.31(m, 1 \mathrm{H}, \mathrm{Ar}-\mathrm{H}) ; 7.02-6.97(m, 2 \mathrm{H}$, Ar-H); $5.25(d d, 1 \mathrm{H}, J=2.36,2.40 \mathrm{~Hz}, \mathrm{CH}) ; 2.79(d d, 1 \mathrm{H}, J=$ $\left.12.10,12.0 \mathrm{~Hz}, \mathrm{CH}_{2}\right) ; 2.51\left(s, 1 \mathrm{H}, \mathrm{CH}_{2}\right) \cdot{ }^{13} \mathrm{C}$ NMR $(300 \mathrm{MHz}$, DMSO- $\left.d_{6}\right), \delta$ ppm: $178.84 ; 156.71 ; 141.71 ; 138.79 ; 132.76$; 131.24; 128.44; 128.27; 125.49; 121.48; 120.10; 117.47; 75.41; 31.83. Analysis calculated for $\mathrm{C}_{16} \mathrm{H}_{14} \mathrm{~N}_{3} \mathrm{OSCl}$ : $\mathrm{C}, 57.91 ; \mathrm{H}, 4.25$; N, 12.66; S, 9.66. Found: C, 57.85; H, 4.28; N, 12.61; S, 9.59.

\section{Refinement}

Crystal data, data collection and structure refinement details are summarized in Table 2. The $\mathrm{NH}$ and $\mathrm{NH}_{2} \mathrm{H}$ atoms were 
located in a difference-Fourier map and refined freely. The Cbound $\mathrm{H}$ atoms were included in calculated positions and treated as riding atoms: $\mathrm{C}-\mathrm{H}=0.93-0.98 \AA$ with $U_{\text {iso }}(\mathrm{H})=$ $1.2 U_{\mathrm{eq}}(\mathrm{C})$.

\section{Acknowledgements}

We thank Nagaland University, AMRC- IIT Mandi and the University of Hyderabad for the research facilities.

\section{Funding information}

PM is grateful to the SERB-DST, Govt. of India for financial support (grant No. SB/EMEQ-030/2014). RZ also thanks the SERB-DST for financial support.

\section{References}

Andersen, C. L., Jensen, C. S., Mackeprang, K., Du, L., Jørgensen, S. \& Kjaergaard, H. G. (2014). J. Phys. Chem. A, 118, 11074-11082.

Bai, J., Wang, R.-H., Qiao, Y., Wang, A. \& Fang, C.-J. (2017). Drug Des. Dev. Ther. 11, 2227-2237.

Bargale, S. \& Shastry, V. R. (1988). Orient. J. Chem. 4, 53-57.

Bargujar, S., Chandra, S., Chauhan, R., Rajor, H. K. \& Bhardwaj, J. (2018). Appl. Organomet. Chem. 32, e4149-e4162.

Bauvois, B., Puiffe, M.-L., Bongui, J.-B., Paillat, S., Monneret, C. \& Dauzonne, D. (2003). J. Med. Chem. 46, 3900-3913.

Brodowska, K., Sykuła, A., Garribba, E., Łodyga-Chruścińska, E. \& Sójka, M. (2016). Transition Met. Chem. 41, 179-189.

Dolomanov, O. V., Bourhis, L. J., Gildea, R. J., Howard, J. A. K. \& Puschmann, H. (2009). J. Appl. Cryst. 42, 339-341.

Groom, C. R., Bruno, I. J., Lightfoot, M. P. \& Ward, S. C. (2016). Acta Cryst. B72, 171-179.

Macrae, C. F., Bruno, I. J., Chisholm, J. A., Edgington, P. R., McCabe, P., Pidcock, E., Rodriguez-Monge, L., Taylor, R., van de Streek, J. \& Wood, P. A. (2008). J. Appl. Cryst. 41, 466-470.

McKinnon, J. J., Jayatilaka, D. \& Spackman, M. A. (2007). Chem. Commun. pp. 3814-3816.

Nie, A., Ghosh, S. \& Huang, Z. (2006). Acta Cryst. E62, o1824-o1825.

Rigaku OD (2015). CrysAlis PRO. Rigaku Oxford Diffraction, Yarnton, England.

Sheldrick, G. M. (2015a). Acta Cryst. A71, 3-8.

Sheldrick, G. M. (2015b). Acta Cryst. C71, 3-8.

Spackman, M. A. \& Jayatilaka, D. (2009). CrystEngComm, 11, 19-32. Spek, A. L. (2009). Acta Cryst. D65, 148-155.

Tan, S. L., Jotani, M. M. \& Tiekink, E. R. T. (2019). Acta Cryst. E75, 308-318.
Table 2

Experimental details.

\begin{tabular}{|c|c|}
\hline \multicolumn{2}{|l|}{ Crystal data } \\
\hline Chemical formula & $\mathrm{C}_{16} \mathrm{H}_{14} \mathrm{ClN}_{3} \mathrm{OS}$ \\
\hline$M_{\mathrm{r}}$ & 331.81 \\
\hline Crystal system, space group & Triclinic, $P \overline{1}$ \\
\hline Temperature $(\mathrm{K})$ & 293 \\
\hline$a, b, c(\AA)$ & $7.8218(7), 8.4207(6), 12.3402(11)$ \\
\hline$\alpha, \beta, \gamma\left({ }^{\circ}\right)$ & $99.838(7), 95.771(7), 96.515(7)$ \\
\hline$V\left(\AA^{3}\right)$ & $789.66(12)$ \\
\hline$Z$ & 2 \\
\hline Radiation type & $\mathrm{Cu} K \alpha$ \\
\hline$\mu\left(\mathrm{mm}^{-1}\right)$ & 3.41 \\
\hline Crystal size $(\mathrm{mm})$ & $0.50 \times 0.17 \times 0.10$ \\
\hline \multicolumn{2}{|l|}{ Data collection } \\
\hline Diffractometer & $\begin{array}{l}\text { Rigaku OD, SuperNova, Dual, Cu } \\
\text { at zero, Eos }\end{array}$ \\
\hline Absorption correction & $\begin{array}{l}\text { Gaussian (CrysAlis PRO; Rigaku } \\
\text { OD, 2015) }\end{array}$ \\
\hline$T_{\min }, T_{\max }$ & $0.464,1.000$ \\
\hline $\begin{array}{l}\text { No. of measured, independent and } \\
\text { observed }[I>2 \sigma(I)] \text { reflections }\end{array}$ & $4478,2766,2346$ \\
\hline$R_{\mathrm{int}}$ & 0.019 \\
\hline$(\sin \theta / \lambda)_{\max }\left(\AA^{-1}\right)$ & 0.596 \\
\hline \multicolumn{2}{|l|}{ Refinement } \\
\hline$R\left[F^{2}>2 \sigma\left(F^{2}\right)\right], w R\left(F^{2}\right), S$ & $0.042,0.121,1.05$ \\
\hline No. of reflections & 2766 \\
\hline No. of parameters & 211 \\
\hline $\mathrm{H}$-atom treatment & $\begin{array}{l}\mathrm{H} \text { atoms treated by a mixture of } \\
\text { independent and constrained } \\
\text { refinement }\end{array}$ \\
\hline$\Delta \rho_{\max }, \Delta \rho_{\min }\left(\mathrm{e} \AA^{-3}\right)$ & $0.51,-0.41$ \\
\hline
\end{tabular}

Computer programs: CrysAlis PRO (Rigaku OD, 2015), SHELXT (Sheldrick, 2015a), SHELXL2018/03 (Sheldrick, 2015b), OLEX2 (Dolomanov et al, 2009), Mercury (Macrae et al., 2008), SHELXL2018/03 (Sheldrick, 2015b), PLATON (Spek, 2009) and publCIF (Westrip, 2010).

Testai, L. \& Calderone, V. (2017). Nutrients 9, 502-514.

Turner, M. J., McKinnon, J. J., Wolff, S. K., Grimwood, D. J., Spackman, P. R., Jayatilaka, D. \& Spackman, M. A. (2017). CrystalExplorer17. University of Western Australia. http://hirshfeldsurface.net

Umamatheswari, S., Pratha, J. J. \& Kabilan, S. (2011). J. Mol. Struct. 989, 1-9.

Walters, M. A., Roche, C. L., Rheingold, A. L. \& Kassel, S. W. (2005). Inorg. Chem. 44, 3777-3779.

Westrip, S. P. (2010). J. Appl. Cryst. 43, 920-925.

Zheng, X., Jiang, H., Xie, J., Yin, Z. \& Zhang, H. (2013). Synth. Commun. 43, 1023-1029. 


\section{supporting information}

Acta Cryst. (2019). E75, 707-710 [https://doi.org/10.1107/S2056989019005073]

Crystal structure and Hirshfeld surface analysis of rac-2-[2-(4-chlorophenyl)-3,4-dihydro-2H-1-benzopyran-4-ylidene]hydrazine-1-carbothioamide

Ruokuosenuo Zatsu, Prabhakar Maddela, M. Indira Devi, Ranjit Singh and Chullikkattil P. Pradeep

Computing details

Data collection: CrysAlis PRO (Rigaku OD, 2015); cell refinement: CrysAlis PRO (Rigaku OD, 2015); data reduction: CrysAlis PRO (Rigaku OD, 2015); program(s) used to solve structure: SHELXT (Sheldrick, 2015a); program(s) used to refine structure: SHELXL2018/03 (Sheldrick, 2015b); molecular graphics: OLEX2 (Dolomanov et al., 2009) and Mercury (Macrae et al., 2008); software used to prepare material for publication: OLEX2 (Dolomanov et al., 2009), SHELXL2018/03 (Sheldrick, 2015b), PLATON (Spek, 2009) and publCIF (Westrip, 2010). rac-2-[2-(4-Chlorophenyl)-3,4-dihydro-2H-1-benzopyran-4-ylidene] hydrazine-1-carbothioamide

Crystal data

$\mathrm{C}_{16} \mathrm{H}_{14} \mathrm{ClN}_{3} \mathrm{OS}$

$M_{r}=331.81$

Triclinic, $P \overline{1}$

$a=7.8218(7) \AA$

$b=8.4207(6) \AA$

$c=12.3402(11) \AA$

$\alpha=99.838(7)^{\circ}$

$\beta=95.771(7)^{\circ}$

$\gamma=96.515(7)^{\circ}$

$V=789.66(12) \AA^{3}$

Data collection

Rigaku OD, SuperNova, Dual, $\mathrm{Cu}$ at zero, Eos diffractometer

Radiation source: micro-focus sealed X-ray tube $\omega$ scans

Absorption correction: gaussian

(CrysAlis PRO; Rigaku OD, 2015)

$T_{\min }=0.464, T_{\max }=1.000$

4478 measured reflections

\section{Refinement}

Refinement on $F^{2}$

Least-squares matrix: full

$R\left[F^{2}>2 \sigma\left(F^{2}\right)\right]=0.042$

$w R\left(F^{2}\right)=0.121$

$S=1.05$

2766 reflections
$Z=2$

$F(000)=344$

$D_{\mathrm{x}}=1.395 \mathrm{Mg} \mathrm{m}^{-3}$

$\mathrm{Cu} K \alpha$ radiation, $\lambda=1.54184 \AA$

Cell parameters from 2014 reflections

$\theta=3.7-66.5^{\circ}$

$\mu=3.41 \mathrm{~mm}^{-1}$

$T=293 \mathrm{~K}$

Plate, yellow

$0.50 \times 0.17 \times 0.10 \mathrm{~mm}$

2766 independent reflections

2346 reflections with $I>2 \sigma(I)$

$R_{\text {int }}=0.019$

$\theta_{\text {max }}=66.7^{\circ}, \theta_{\min }=3.7^{\circ}$

$h=-9 \rightarrow 9$

$k=-10 \rightarrow 7$

$l=-14 \rightarrow 14$

211 parameters

0 restraints

Primary atom site location: structure-invariant direct methods

Secondary atom site location: difference Fourier map 
Hydrogen site location: mixed

$\mathrm{H}$ atoms treated by a mixture of independent and constrained refinement

$$
\begin{aligned}
& w=1 /\left[\sigma^{2}\left(F_{\mathrm{o}}^{2}\right)+(0.0655 P)^{2}+0.1825 P\right] \\
& \text { where } P=\left(F_{\mathrm{o}}^{2}+2 F_{\mathrm{c}}^{2}\right) / 3 \\
& (\Delta / \sigma)_{\max }=0.001 \\
& \Delta \rho_{\max }=0.51 \text { e } \AA^{-3} \\
& \Delta \rho_{\min }=-0.41 \mathrm{e} \AA^{-3}
\end{aligned}
$$

Special details

Geometry. All esds (except the esd in the dihedral angle between two 1.s. planes) are estimated using the full covariance

\begin{tabular}{|c|c|c|c|c|}
\hline & $x$ & $y$ & $z$ & $U_{\text {iso }} * / U_{\text {eq }}$ \\
\hline $\mathrm{S} 1$ & $0.03040(9)$ & $0.27189(6)$ & $1.04983(4)$ & $0.0560(2)$ \\
\hline $\mathrm{Cl1}$ & $0.63580(11)$ & $1.37767(8)$ & $0.85742(7)$ & 0.0909 (3) \\
\hline $\mathrm{O} 1$ & $0.2996(2)$ & $0.65276(19)$ & $0.57103(12)$ & $0.0597(4)$ \\
\hline N1 & $0.1205(2)$ & $0.3125(2)$ & $0.75055(14)$ & $0.0492(4)$ \\
\hline $\mathrm{N} 2$ & $0.1066(3)$ & $0.3537(2)$ & $0.86177(14)$ & $0.0492(4)$ \\
\hline $\mathrm{H} 2 \mathrm{~N}$ & $0.089(3)$ & $0.448(3)$ & $0.892(2)$ & $0.061(7)^{*}$ \\
\hline N3 & $0.0872(4)$ & 0.0835 & $0.86608(18)$ & $0.0689(6)$ \\
\hline H3AN & $0.115(4)$ & $0.073(3)$ & $0.800(3)$ & $0.076(9)^{*}$ \\
\hline $\mathrm{H} 3 \mathrm{BN}$ & $0.062(3)$ & -0.002 & $0.896(2)$ & $0.074(8)^{*}$ \\
\hline $\mathrm{C} 2$ & 0.3685 & $0.6714(3)$ & $0.68520(18)$ & $0.0499(5)$ \\
\hline $\mathrm{H} 2$ & 0.467722 & 0.610398 & 0.689533 & $0.060 *$ \\
\hline $\mathrm{C} 3$ & 0.2332 & 0.6009 & $0.74971(17)$ & $0.0493(5)$ \\
\hline $\mathrm{H} 3 \mathrm{~A}$ & 0.281782 & 0.610110 & 0.826354 & $0.059 *$ \\
\hline H3B & 0.134816 & 0.661355 & 0.748204 & $0.059 *$ \\
\hline $\mathrm{C} 4$ & $0.1747(3)$ & $0.4250(2)$ & $0.69944(17)$ & $0.0450(4)$ \\
\hline $\mathrm{C} 5$ & $0.1826(3)$ & $0.3784(3)$ & $0.58006(16)$ & $0.0474(5)$ \\
\hline C6 & 0.1305 & 0.2204 & 0.52088 (19) & $0.0606(6)$ \\
\hline H6 & 0.091678 & 0.139229 & 0.558488 & $0.073 *$ \\
\hline $\mathrm{C} 7$ & 0.1353 & $0.1822(3)$ & $0.4087(2)$ & $0.0681(7)$ \\
\hline $\mathrm{H} 7$ & 0.104231 & 0.075412 & 0.371456 & $0.082^{*}$ \\
\hline $\mathrm{C} 8$ & $0.1866(3)$ & $0.3031(4)$ & $0.35112(19)$ & $0.0650(7)$ \\
\hline H8 & 0.186752 & 0.278143 & 0.274751 & $0.078^{*}$ \\
\hline C9 & 0.2373 & 0.4599 & 0.40644 (19) & $0.0607(6)$ \\
\hline H9 & 0.269457 & 0.541354 & 0.367367 & $0.073^{*}$ \\
\hline $\mathrm{C} 10$ & $0.2406(3)$ & $0.4967(3)$ & $0.52061(17)$ & $0.0500(5)$ \\
\hline C11 & 0.4330 & 0.8494 & $0.72737(18)$ & $0.0506(5)$ \\
\hline $\mathrm{C} 12$ & 0.4111 & $0.9656(3)$ & $0.6620(2)$ & $0.0604(6)$ \\
\hline H12 & 0.352887 & 0.934776 & 0.590588 & $0.073^{*}$ \\
\hline $\mathrm{C} 13$ & $0.4752(3)$ & $1.1273(3)$ & $0.7020(2)$ & $0.0668(7)$ \\
\hline H13 & 0.460876 & 1.204685 & 0.657535 & $0.080^{*}$ \\
\hline $\mathrm{C} 14$ & $0.5595(3)$ & $1.1727(3)$ & $0.8070(2)$ & $0.0624(6)$ \\
\hline C15 & $0.5862(4)$ & $1.0597(3)$ & $0.8729(2)$ & $0.0719(7)$ \\
\hline H15 & 0.645873 & 1.091518 & 0.943806 & $0.086^{*}$ \\
\hline C16 & $0.5233(4)$ & 0.8983 & $0.8325(2)$ & $0.0682(7)$ \\
\hline
\end{tabular}
matrix. The cell esds are taken into account individually in the estimation of esds in distances, angles and torsion angles; correlations between esds in cell parameters are only used when they are defined by crystal symmetry. An approximate (isotropic) treatment of cell esds is used for estimating esds involving 1.s. planes.

Fractional atomic coordinates and isotropic or equivalent isotropic displacement parameters $\left(\AA^{2}\right)$ 
supporting information

\begin{tabular}{lllll} 
H16 & 0.541890 & 0.821123 & 0.876540 & $0.082^{*}$ \\
C17 & $0.0759(3)$ & $0.2312(2)$ & $0.91799(17)$ & $0.0470(5)$ \\
\hline
\end{tabular}

Atomic displacement parameters $\left(\AA^{2}\right)$

\begin{tabular}{lllllll}
\hline & $U^{11}$ & $U^{22}$ & $U^{33}$ & $U^{12}$ & $U^{13}$ & $U^{23}$ \\
\hline S1 & $0.0973(4)$ & $0.0372(3)$ & $0.0360(3)$ & $0.0047(3)$ & $0.0169(3)$ & $0.0114(2)$ \\
C11 & $0.1088(6)$ & $0.0550(4)$ & $0.1009(6)$ & $-0.0103(4)$ & $-0.0092(4)$ & $0.0184(4)$ \\
O1 & $0.0846(11)$ & $0.0556(9)$ & $0.0446(8)$ & $0.0059(8)$ & $0.0172(7)$ & $0.0219(7)$ \\
N1 & $0.0673(11)$ & $0.0448(9)$ & $0.0385(9)$ & $0.0057(8)$ & $0.0134(8)$ & $0.0132(7)$ \\
N2 & $0.0761(12)$ & $0.0362(9)$ & $0.0386(9)$ & $0.0049(8)$ & $0.0174(8)$ & $0.0121(7)$ \\
N3 & $0.127(2)$ & $0.0374(10)$ & $0.0482(12)$ & $0.0088(11)$ & $0.0328(12)$ & $0.0121(9)$ \\
C2 & $0.0547(11)$ & $0.0521(12)$ & $0.0491(12)$ & $0.0100(9)$ & $0.0147(9)$ & $0.0200(9)$ \\
C3 & $0.0593(12)$ & $0.0485(11)$ & $0.0438(11)$ & $0.0050(9)$ & $0.0130(9)$ & $0.0163(9)$ \\
C4 & $0.0498(11)$ & $0.0480(11)$ & $0.0406(10)$ & $0.0083(8)$ & $0.0092(8)$ & $0.0143(8)$ \\
C5 & $0.0500(11)$ & $0.0577(12)$ & $0.0380(10)$ & $0.0090(9)$ & $0.0085(8)$ & $0.0154(9)$ \\
C6 & $0.0730(15)$ & $0.0630(14)$ & $0.0427(12)$ & $-0.0017(11)$ & $0.0043(10)$ & $0.0101(10)$ \\
C7 & $0.0738(16)$ & $0.0771(17)$ & $0.0467(13)$ & $0.0005(13)$ & $0.0025(11)$ & $0.0017(12)$ \\
C8 & $0.0578(13)$ & $0.101(2)$ & $0.0356(11)$ & $0.0103(13)$ & $0.0068(9)$ & $0.0095(12)$ \\
C9 & $0.0627(13)$ & $0.0833(17)$ & $0.0434(12)$ & $0.0128(12)$ & $0.0161(10)$ & $0.0244(11)$ \\
C10 & $0.0519(11)$ & $0.0594(13)$ & $0.0440(11)$ & $0.0128(9)$ & $0.0121(9)$ & $0.0173(9)$ \\
C11 & $0.0518(11)$ & $0.0508(12)$ & $0.0546(12)$ & $0.0066(9)$ & $0.0154(9)$ & $0.0198(10)$ \\
C12 & $0.0671(14)$ & $0.0556(13)$ & $0.0624(14)$ & $0.0069(10)$ & $0.0047(11)$ & $0.0241(11)$ \\
C13 & $0.0729(15)$ & $0.0549(14)$ & $0.0773(17)$ & $0.0061(11)$ & $0.0021(13)$ & $0.0305(12)$ \\
C14 & $0.0617(13)$ & $0.0518(13)$ & $0.0755(16)$ & $0.0005(10)$ & $0.0090(12)$ & $0.0208(11)$ \\
C15 & $0.0828(17)$ & $0.0661(16)$ & $0.0636(15)$ & $-0.0061(13)$ & $-0.0029(13)$ & $0.0199(12)$ \\
C16 & $0.0828(17)$ & $0.0581(14)$ & $0.0663(16)$ & $0.0004(12)$ & $0.0026(13)$ & $0.0283(12)$ \\
C17 & $0.0642(12)$ & $0.0382(10)$ & $0.0407(10)$ & $0.0027(9)$ & $0.0109(9)$ & $0.0129(8)$ \\
& & & & & &
\end{tabular}

Geometric parameters $\left(\AA,{ }^{\circ}\right)$

\begin{tabular}{llll}
\hline $\mathrm{S} 1-\mathrm{C} 17$ & $1.687(2)$ & $\mathrm{C} 5-\mathrm{C} 6$ & $1.398(3)$ \\
$\mathrm{C} 11-\mathrm{C} 14$ & $1.746(2)$ & $\mathrm{C} 6-\mathrm{C} 7$ & $1.372(3)$ \\
$\mathrm{O} 1-\mathrm{C} 10$ & $1.361(3)$ & $\mathrm{C} 6-\mathrm{H} 6$ & 0.9300 \\
$\mathrm{O} 1-\mathrm{C} 2$ & $1.433(3)$ & $\mathrm{C} 7-\mathrm{C} 8$ & $1.383(4)$ \\
$\mathrm{N} 1-\mathrm{C} 4$ & $1.281(3)$ & $\mathrm{C} 7-\mathrm{H} 7$ & 0.9300 \\
$\mathrm{~N} 1-\mathrm{N} 2$ & $1.375(2)$ & $\mathrm{C} 8-\mathrm{C} 9$ & $1.373(4)$ \\
$\mathrm{N} 2-\mathrm{C} 17$ & $1.351(3)$ & $\mathrm{C} 8-\mathrm{H} 8$ & 0.9300 \\
$\mathrm{~N} 2-\mathrm{H} 2 \mathrm{~N}$ & $0.85(3)$ & $\mathrm{C} 9-\mathrm{C} 10$ & $1.387(3)$ \\
$\mathrm{N} 3-\mathrm{C} 17$ & $1.315(3)$ & $\mathrm{C} 9-\mathrm{H} 9$ & 0.9300 \\
$\mathrm{~N} 3-\mathrm{H} 3 \mathrm{AN}$ & $0.85(3)$ & $\mathrm{C} 11-\mathrm{C} 16$ & $1.385(3)$ \\
$\mathrm{N} 3-\mathrm{H} 3 \mathrm{BN}$ & $0.88(3)$ & $\mathrm{C} 11-\mathrm{C} 12$ & $1.385(3)$ \\
$\mathrm{C} 2-\mathrm{C} 11$ & $1.511(3)$ & $\mathrm{C} 12-\mathrm{C} 13$ & $1.383(3)$ \\
$\mathrm{C} 2-\mathrm{C} 3$ & $1.514(3)$ & $\mathrm{C} 12-\mathrm{H} 12$ & 0.9300 \\
$\mathrm{C} 2-\mathrm{H} 2$ & 0.9800 & $\mathrm{C} 13-\mathrm{C} 14$ & $1.364(4)$ \\
$\mathrm{C} 3-\mathrm{C} 4$ & $1.505(3)$ & $\mathrm{C} 13-\mathrm{H} 13$ & 0.9300 \\
$\mathrm{C} 3-\mathrm{H} 3 \mathrm{~A}$ & 0.9700 & $\mathrm{C} 14-\mathrm{C} 15$ & $1.374(4)$ \\
$\mathrm{C} 3-\mathrm{H} 3 \mathrm{~B}$ & 0.9700 & $\mathrm{C} 15-\mathrm{C} 16$ & $1.381(4)$
\end{tabular}




\begin{tabular}{|c|c|c|c|}
\hline $\mathrm{C} 4-\mathrm{C} 5$ & $1.468(3)$ & C15-H15 & 0.9300 \\
\hline $\mathrm{C} 5-\mathrm{C} 10$ & $1.396(3)$ & $\mathrm{C} 16-\mathrm{H} 16$ & 0.9300 \\
\hline $\mathrm{C} 10-\mathrm{O} 1-\mathrm{C} 2$ & $114.56(16)$ & $\mathrm{C} 8-\mathrm{C} 7-\mathrm{H} 7$ & 120.1 \\
\hline $\mathrm{C} 4-\mathrm{N} 1-\mathrm{N} 2$ & $118.41(17)$ & $\mathrm{C} 9-\mathrm{C} 8-\mathrm{C} 7$ & $120.2(2)$ \\
\hline $\mathrm{C} 17-\mathrm{N} 2-\mathrm{N} 1$ & $117.54(17)$ & $\mathrm{C} 9-\mathrm{C} 8-\mathrm{H} 8$ & 119.9 \\
\hline $\mathrm{C} 17-\mathrm{N} 2-\mathrm{H} 2 \mathrm{~N}$ & $117.8(17)$ & $\mathrm{C} 7-\mathrm{C} 8-\mathrm{H} 8$ & 119.9 \\
\hline $\mathrm{N} 1-\mathrm{N} 2-\mathrm{H} 2 \mathrm{~N}$ & $122.4(17)$ & $\mathrm{C} 8-\mathrm{C} 9-\mathrm{C} 10$ & $120.0(2)$ \\
\hline $\mathrm{C} 17-\mathrm{N} 3-\mathrm{H} 3 \mathrm{AN}$ & $117(2)$ & $\mathrm{C} 8-\mathrm{C} 9-\mathrm{H} 9$ & 120.0 \\
\hline $\mathrm{C} 17-\mathrm{N} 3-\mathrm{H} 3 \mathrm{BN}$ & $121.9(18)$ & $\mathrm{C} 10-\mathrm{C} 9-\mathrm{H} 9$ & 120.0 \\
\hline $\mathrm{H} 3 \mathrm{AN}-\mathrm{N} 3-\mathrm{H} 3 \mathrm{BN}$ & $121(3)$ & $\mathrm{O} 1-\mathrm{C} 10-\mathrm{C} 9$ & $117.1(2)$ \\
\hline $\mathrm{O} 1-\mathrm{C} 2-\mathrm{C} 11$ & $107.89(17)$ & $\mathrm{O} 1-\mathrm{C} 10-\mathrm{C} 5$ & $121.98(19)$ \\
\hline $\mathrm{O} 1-\mathrm{C} 2-\mathrm{C} 3$ & $110.01(18)$ & $\mathrm{C} 9-\mathrm{C} 10-\mathrm{C} 5$ & $120.9(2)$ \\
\hline $\mathrm{C} 11-\mathrm{C} 2-\mathrm{C} 3$ & $114.02(18)$ & $\mathrm{C} 16-\mathrm{C} 11-\mathrm{C} 12$ & $118.5(2)$ \\
\hline $\mathrm{O} 1-\mathrm{C} 2-\mathrm{H} 2$ & 108.3 & $\mathrm{C} 16-\mathrm{C} 11-\mathrm{C} 2$ & $119.6(2)$ \\
\hline $\mathrm{C} 11-\mathrm{C} 2-\mathrm{H} 2$ & 108.3 & $\mathrm{C} 12-\mathrm{C} 11-\mathrm{C} 2$ & $121.8(2)$ \\
\hline $\mathrm{C} 3-\mathrm{C} 2-\mathrm{H} 2$ & 108.3 & $\mathrm{C} 13-\mathrm{C} 12-\mathrm{C} 11$ & $120.5(2)$ \\
\hline $\mathrm{C} 4-\mathrm{C} 3-\mathrm{C} 2$ & $109.75(17)$ & $\mathrm{C} 13-\mathrm{C} 12-\mathrm{H} 12$ & 119.7 \\
\hline $\mathrm{C} 4-\mathrm{C} 3-\mathrm{H} 3 \mathrm{~A}$ & 109.7 & $\mathrm{C} 11-\mathrm{C} 12-\mathrm{H} 12$ & 119.7 \\
\hline $\mathrm{C} 2-\mathrm{C} 3-\mathrm{H} 3 \mathrm{~A}$ & 109.7 & $\mathrm{C} 14-\mathrm{C} 13-\mathrm{C} 12$ & $119.7(2)$ \\
\hline $\mathrm{C} 4-\mathrm{C} 3-\mathrm{H} 3 \mathrm{~B}$ & 109.7 & $\mathrm{C} 14-\mathrm{C} 13-\mathrm{H} 13$ & 120.1 \\
\hline $\mathrm{C} 2-\mathrm{C} 3-\mathrm{H} 3 \mathrm{~B}$ & 109.7 & $\mathrm{C} 12-\mathrm{C} 13-\mathrm{H} 13$ & 120.1 \\
\hline $\mathrm{H} 3 \mathrm{~A}-\mathrm{C} 3-\mathrm{H} 3 \mathrm{~B}$ & 108.2 & $\mathrm{C} 13-\mathrm{C} 14-\mathrm{C} 15$ & $121.0(2)$ \\
\hline $\mathrm{N} 1-\mathrm{C} 4-\mathrm{C} 5$ & $117.19(19)$ & $\mathrm{C} 13-\mathrm{C} 14-\mathrm{Cl1}$ & $119.26(19)$ \\
\hline $\mathrm{N} 1-\mathrm{C} 4-\mathrm{C} 3$ & $126.53(19)$ & $\mathrm{C} 15-\mathrm{C} 14-\mathrm{Cl} 1$ & $119.7(2)$ \\
\hline $\mathrm{C} 5-\mathrm{C} 4-\mathrm{C} 3$ & $116.28(17)$ & $\mathrm{C} 14-\mathrm{C} 15-\mathrm{C} 16$ & $119.1(3)$ \\
\hline $\mathrm{C} 10-\mathrm{C} 5-\mathrm{C} 6$ & $117.4(2)$ & $\mathrm{C} 14-\mathrm{C} 15-\mathrm{H} 15$ & 120.4 \\
\hline $\mathrm{C} 10-\mathrm{C} 5-\mathrm{C} 4$ & $119.36(19)$ & $\mathrm{C} 16-\mathrm{C} 15-\mathrm{H} 15$ & 120.4 \\
\hline $\mathrm{C} 6-\mathrm{C} 5-\mathrm{C} 4$ & $123.21(19)$ & $\mathrm{C} 15-\mathrm{C} 16-\mathrm{C} 11$ & $121.0(2)$ \\
\hline $\mathrm{C} 7-\mathrm{C} 6-\mathrm{C} 5$ & $121.6(2)$ & $\mathrm{C} 15-\mathrm{C} 16-\mathrm{H} 16$ & 119.5 \\
\hline $\mathrm{C} 7-\mathrm{C} 6-\mathrm{H} 6$ & 119.2 & $\mathrm{C} 11-\mathrm{C} 16-\mathrm{H} 16$ & 119.5 \\
\hline $\mathrm{C} 5-\mathrm{C} 6-\mathrm{H} 6$ & 119.2 & $\mathrm{~N} 3-\mathrm{C} 17-\mathrm{N} 2$ & $117.00(19)$ \\
\hline $\mathrm{C} 6-\mathrm{C} 7-\mathrm{C} 8$ & $119.7(2)$ & $\mathrm{N} 3-\mathrm{C} 17-\mathrm{S} 1$ & $122.99(17)$ \\
\hline $\mathrm{C} 6-\mathrm{C} 7-\mathrm{H} 7$ & 120.1 & $\mathrm{~N} 2-\mathrm{C} 17-\mathrm{S} 1$ & $120.00(16)$ \\
\hline $\mathrm{C} 4-\mathrm{N} 1-\mathrm{N} 2-\mathrm{C} 17$ & $-169.8(2)$ & $\mathrm{C} 8-\mathrm{C} 9-\mathrm{C} 10-\mathrm{C} 5$ & $-4.0(3)$ \\
\hline $\mathrm{C} 10-\mathrm{O} 1-\mathrm{C} 2-\mathrm{C} 11$ & $178.22(17)$ & $\mathrm{C} 6-\mathrm{C} 5-\mathrm{C} 10-\mathrm{O} 1$ & $-177.7(2)$ \\
\hline $\mathrm{C} 10-\mathrm{O} 1-\mathrm{C} 2-\mathrm{C} 3$ & $-56.8(2)$ & $\mathrm{C} 4-\mathrm{C} 5-\mathrm{C} 10-\mathrm{O} 1$ & $3.8(3)$ \\
\hline $\mathrm{O} 1-\mathrm{C} 2-\mathrm{C} 3-\mathrm{C} 4$ & $57.2(2)$ & $\mathrm{C} 6-\mathrm{C} 5-\mathrm{C} 10-\mathrm{C} 9$ & $3.4(3)$ \\
\hline $\mathrm{C} 11-\mathrm{C} 2-\mathrm{C} 3-\mathrm{C} 4$ & $178.59(17)$ & $\mathrm{C} 4-\mathrm{C} 5-\mathrm{C} 10-\mathrm{C} 9$ & $-175.0(2)$ \\
\hline $\mathrm{N} 2-\mathrm{N} 1-\mathrm{C} 4-\mathrm{C} 5$ & $-178.00(17)$ & $\mathrm{O} 1-\mathrm{C} 2-\mathrm{C} 11-\mathrm{C} 16$ & $-173.0(2)$ \\
\hline $\mathrm{N} 2-\mathrm{N} 1-\mathrm{C} 4-\mathrm{C} 3$ & $2.7(3)$ & $\mathrm{C} 3-\mathrm{C} 2-\mathrm{C} 11-\mathrm{C} 16$ & $64.5(3)$ \\
\hline $\mathrm{C} 2-\mathrm{C} 3-\mathrm{C} 4-\mathrm{N} 1$ & $150.2(2)$ & $\mathrm{O} 1-\mathrm{C} 2-\mathrm{C} 11-\mathrm{C} 12$ & $4.2(3)$ \\
\hline $\mathrm{C} 2-\mathrm{C} 3-\mathrm{C} 4-\mathrm{C} 5$ & $-29.1(2)$ & $\mathrm{C} 3-\mathrm{C} 2-\mathrm{C} 11-\mathrm{C} 12$ & $-118.3(2)$ \\
\hline $\mathrm{N} 1-\mathrm{C} 4-\mathrm{C} 5-\mathrm{C} 10$ & $179.93(19)$ & $\mathrm{C} 16-\mathrm{C} 11-\mathrm{C} 12-\mathrm{C} 13$ & $-1.3(4)$ \\
\hline $\mathrm{C} 3-\mathrm{C} 4-\mathrm{C} 5-\mathrm{C} 10$ & $-0.7(3)$ & $\mathrm{C} 2-\mathrm{C} 11-\mathrm{C} 12-\mathrm{C} 13$ & $-178.5(2)$ \\
\hline $\mathrm{N} 1-\mathrm{C} 4-\mathrm{C} 5-\mathrm{C} 6$ & $1.6(3)$ & $\mathrm{C} 11-\mathrm{C} 12-\mathrm{C} 13-\mathrm{C} 14$ & $-0.5(4)$ \\
\hline $\mathrm{C} 3-\mathrm{C} 4-\mathrm{C} 5-\mathrm{C} 6$ & $-179.1(2)$ & $\mathrm{C} 12-\mathrm{C} 13-\mathrm{C} 14-\mathrm{C} 15$ & $1.8(4)$ \\
\hline
\end{tabular}




$\begin{array}{llll}\mathrm{C} 10-\mathrm{C} 5-\mathrm{C} 6-\mathrm{C} 7 & -0.2(4) & \mathrm{C} 12-\mathrm{C} 13-\mathrm{C} 14-\mathrm{C} 11 & -178.7(2) \\ \mathrm{C} 4-\mathrm{C} 5-\mathrm{C} 6-\mathrm{C} 7 & 178.2(2) & \mathrm{C} 13-\mathrm{C} 14-\mathrm{C} 15-\mathrm{C} 16 & -1.3(4) \\ \mathrm{C} 5-\mathrm{C} 6-\mathrm{C} 7-\mathrm{C} 8 & -2.4(4) & \mathrm{C} 11-\mathrm{C} 14-\mathrm{C} 15-\mathrm{C} 16 & 179.2(2) \\ \mathrm{C} 6-\mathrm{C} 7-\mathrm{C} 8-\mathrm{C} 9 & 1.9(4) & \mathrm{C} 14-\mathrm{C} 15-\mathrm{C} 16-\mathrm{C} 11 & -0.5(4) \\ \mathrm{C} 7-\mathrm{C} 8-\mathrm{C} 9-\mathrm{C} 10 & 1.3(4) & \mathrm{C} 12-\mathrm{C} 11-\mathrm{C} 16-\mathrm{C} 15 & 1.8(4) \\ \mathrm{C} 2-\mathrm{O} 1-\mathrm{C} 10-\mathrm{C} 9 & -155.12(19) & \mathrm{C} 2-\mathrm{C} 11-\mathrm{C} 16-\mathrm{C} 15 & 179.1(2) \\ \mathrm{C} 2-\mathrm{O} 1-\mathrm{C} 10-\mathrm{C} 5 & 26.0(3) & \mathrm{N} 1-\mathrm{N} 2-\mathrm{C} 17-\mathrm{N} 3 & 9.8(3) \\ \mathrm{C} 8-\mathrm{C} 9-\mathrm{C} 10-\mathrm{O} 1 & 177.1(2) & \mathrm{N} 1-\mathrm{N} 2-\mathrm{C} 17-\mathrm{S} 1 & -171.68(15)\end{array}$

Hydrogen-bond geometry $\left(A,{ }^{\circ}\right)$

\begin{tabular}{lllll}
\hline$D-\mathrm{H} \cdots A$ & $D-\mathrm{H}$ & $\mathrm{H} \cdots A$ & $D \cdots A$ & $D-\mathrm{H} \cdots A$ \\
\hline $\mathrm{N} 2-\mathrm{H} 2 N \cdots \mathrm{S} 1^{\mathrm{i}}$ & $0.85(3)$ & $2.65(3)$ & $3.480(2)$ & $167(2)$ \\
$\mathrm{N} 3-\mathrm{H} 3 B N \cdots \mathrm{S} 1^{\mathrm{ii}}$ & $0.88(3)$ & $2.52(3)$ & $3.392(2)$ & $171(2)$ \\
\hline
\end{tabular}

Symmetry codes: (i) $-x,-y+1,-z+2$; (ii) $-x,-y,-z+2$. 